\title{
AN EYE GAZE DETECTION USING LOW RESOLUTION WEB CAMERA IN DESKTOP ENVIRONMENT
}

\author{
Mahadak Gopal Mahadeo ${ }^{1}$, Landage Vijay Bapurao ${ }^{2}$, Lohare Nitin Panditrao ${ }^{3}$, Solankar Rasika \\ Sharad $^{4}$ \\ ${ }^{I}$ Student, Computer Engineering Department, DYPCOE, Ambi, Maharashtra, India \\ ${ }^{2}$ Student, Computer Engineering Department, DYPCOE, Ambi, Maharashtra, India \\ ${ }^{3}$ Student, Computer Engineering Department, DYPCOE, Ambi, Maharashtra, India \\ ${ }^{4}$ Student, Computer Engineering Department, DYPCOE, Ambi, Maharashtra, India
}

\begin{abstract}
Purpose of this paper is to detect the focus of the eye on monitor in $X$ and $Y$ coordinates with the help of regular low resolution web camera. We are using regular low resolution web camera to get the results of system which benefits in economical way for development. To achieve the systems goal we are using OpenCV (Open Source Computer Vision) library which is open source library which makes system very economical. System is implemented with the help of Viola Jones algorithm which help to maximize the accuracy. This system helps in human-computer interaction. This system helps the blind peoples to control the various systems.
\end{abstract}

Keywords: Gaze Detection, Voila Jones Algorithm, Haar-Like Features.

\section{INTRODUCTION}

The gaze detection is to detect the center of the eyes on the monitor. The goal is achieved with OpenCV library. OpenCV is open source and image processing library which is compatible with various languages like Python, $\mathrm{C}$ and Java. The system accesses the functions of OpenCV to achieve various tasks.

The system is developed for various applications like space mouse controlling and blind person mouse controlling etc. The systems main focus is to detect users focus on monitor and map it in the $\mathrm{X}$ and Y coordinates in system. The system is divided into various different steps and these are as follows:

- Face extraction

- Eye detection

- Eye center calculation

- Eye center mapping

The face extraction and eye detection uses the Voila Jones technique which is widely used because of its faster processing and accuracy. Voila Jones is having different modules like haar classifier, intragral image, adaboost and cascade classifier. Voila Jones extracts the face from background video and calculates whole face area. Same technique is used for eye calculation which divides the face into two parts. From the two parts it divides the upper part into two parts. From main upper part it calculates the center of lower part and started applying the cascade classifier algorithm. For calculating eye center it finds the center of the square which implemented by haar classifier algorithm. After finding the center it compares with world coordinates and windows coordinates. At last it applies the camshift means algorithm while moving head and eyes. The system runs the all processing in loop.

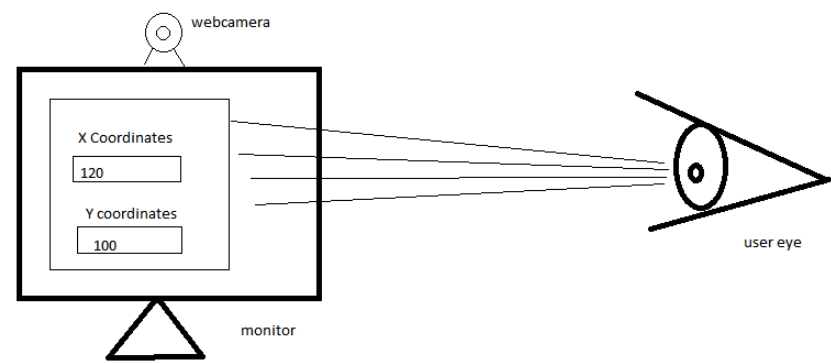

Fig-1: Basic Idea of System

The system uses the multithreading concept because the position of eyes and head changes always runtime and to calculate it requires large processing time on single core. The system takes the help of low resolution web camera to achieve the main goal of system. The camera is of VGA or 0.5-2 megapixels which is having night vision functionality and software with canny edge detector algorithm implemented. The system uses the haar classifier face and eye XML files who is having functions to calculate all extraction and detections.

To track the pilot's eye and develop the correct visual interfaces this system is used in military applications for cockpit [1]. Emotions of person such as anxiety, deceit or hostility can be examined also mental states can be tracked by using eye gaze detection in homeland security applications [2]-[3]. 


\subsection{Existing Methods}

The method of gaze detection on monitor is called Oculography. There are two different methods for gaze estimation [4].

a) Feature-based Gaze Estimation:

This method explores the various characteristics of the human eye to identify the various distinctive features of the eyes like eye corner, eye pupils and corner reflections [5].It has the two methods model based and interpolation based [6].

b) Appearance-based Gaze Estimation:

Based on photometric appearance the appearance based gaze methods detect the gaze position and eyes. It uses the image content to estimate the gaze direction by mapping image data to screen coordinates [7]-[8]. They do not require the calibration of cameras.

\section{PROPOSED METHOD}

Eye gaze detection method contains the eye tracking process which tracks eyes movements with respect to head movements. One of the method capture the eye frames through the web camera 20-30 frames per second and gives it to computer for processing, after produces the results. The video frames help the algorithm for faster processing. Our system uses the OpenCV library which used for HCI (human computer interaction) and mainly meant for real time image processing that contains the functions used in our algorithms.

We use the windows platform with net beans software. First we use the algorithm for face detection then we use it for eyes detection. The algorithm uses haar cascade classifier for face and eyes detection [9]-[10]. Overview of the system is represented by following block diagram:

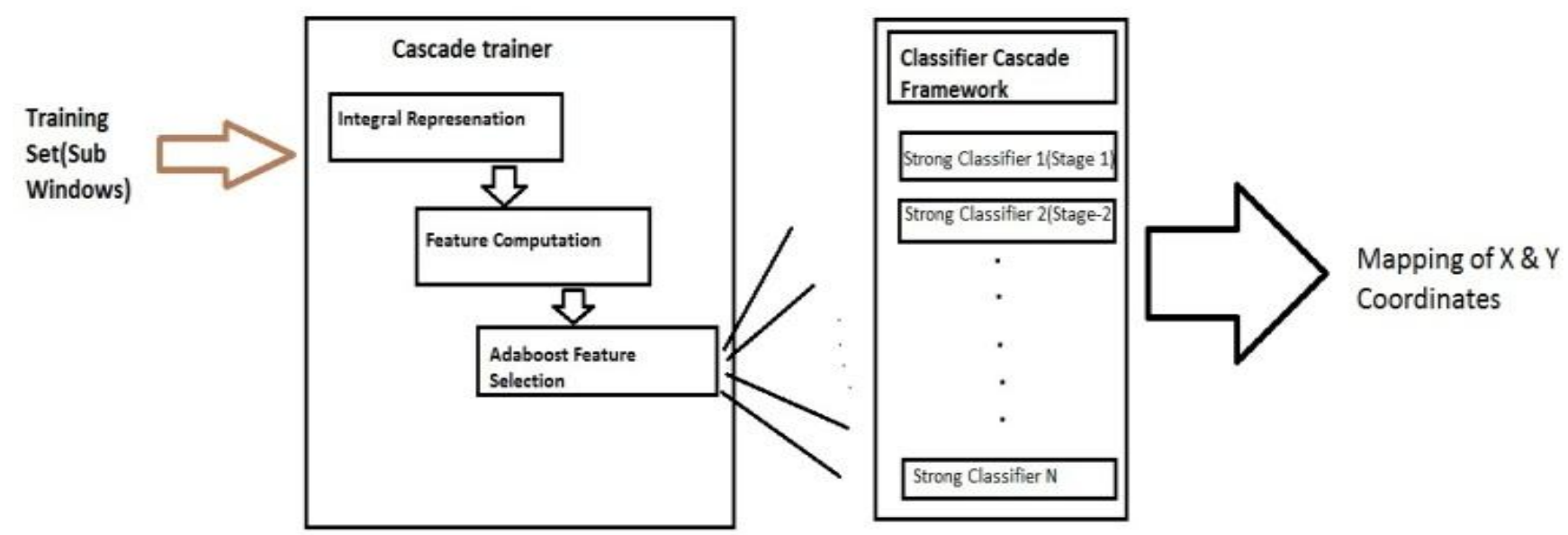

Fig-2: System Architecture

\section{IMPLEMENTATION}

\section{Voila Jones Algorithm:}

This algorithm is used for face and eye detection and it is based on haar features [11]. Haar feature uses the $25 * 25$ rectangular block for feature detection. In it the white portion is subtracted from the grey portion [12].

Following are the haar like features which are commonly used.
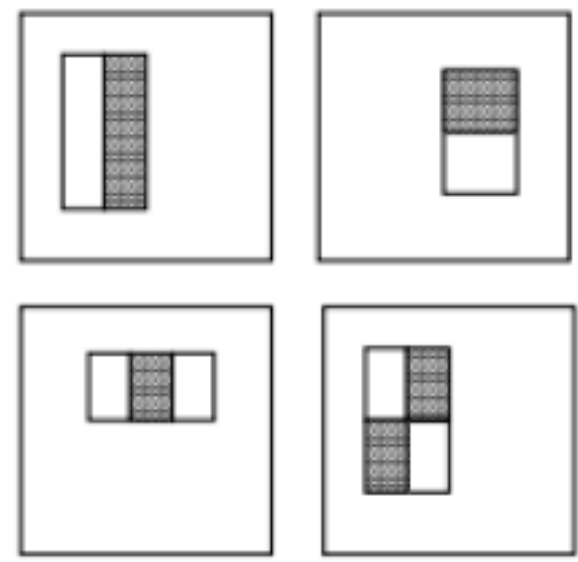

Fig-3: Haar- Like Features
At every stage the face candidates are scanned. The weight of the every feature is calculated by AdaBoost algorithm. Every feature generates the particular value after processing the every rectangle. Each stage has number of values of features in it so AdaBoost algorithm compares the number of stages with values. No face stages values are avoided \& others are forwarded to next stage.

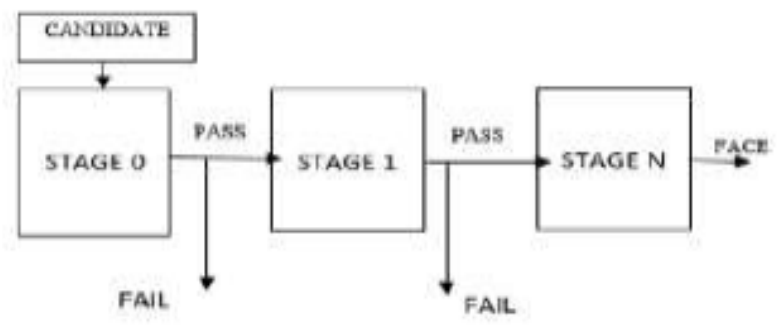

Fig-4: Cascading Stages

From the cascade classifier the features are detected. In this system the following classifiers are used:

- haarcascade_frontalface_at12.xml

- haarcascade_eye.xml 
In system first the face is detected \& then eyes are detected with the help of the area of the face as reference. In OpenCV, there are applications which support the training of a cascade classifier: opencv_haartraining \& opencv_traincascade. The program has following functions

- main() : In the main function we load the XML file \& classifier file.

- detectEye(): In the detectEye function the multiscale eyes $\&$ face are detected.

- trackeye(): In the trackeye function the mapping of coordinates is performed.

\section{CONCLUSIONS AND RESULT:}

By using the Voila Jones technique the accuracy of the system is achieved \& users focus calculated. The system generates the output in the $\mathrm{X} \& \mathrm{Y}$ coordinates. Following is the desired system which is having the circle at eyes centers.

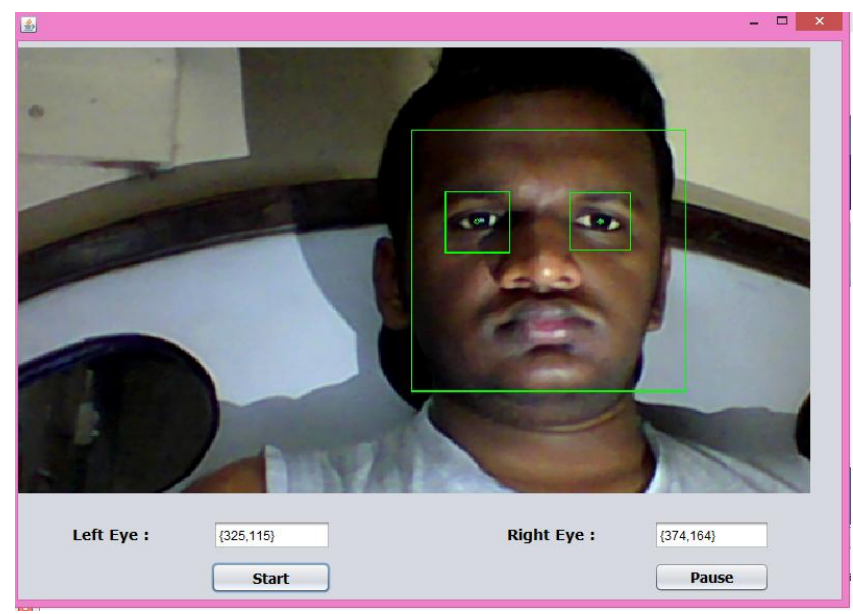

Fig-5: Actual System

The result of the system is represented in the $\mathrm{X}$ and $\mathrm{Y}$ coordinates. Following is the table which shows the accuracy level in the left and right eyes.

\begin{tabular}{|l|l|l|l|}
\hline $\begin{array}{l}\text { Sr. } \\
\text { No. }\end{array}$ & $\begin{array}{l}\text { Left Eye } \\
\text { Coordinates }\end{array}$ & $\begin{array}{l}\text { Right Eye } \\
\text { Coordinates }\end{array}$ & $\begin{array}{l}\text { Accuracy } \\
\text { Achieved }\end{array}$ \\
\hline 1 & $\{325,125\}$ & $\{374,164\}$ & 69 \\
\hline 2 & $\{423,162\}$ & $\{421,136\}$ & 72 \\
\hline 3 & $\{546,215\}$ & $\{521,231\}$ & 71.45 \\
\hline 4 & $\{432,300\}$ & $\{478,321\}$ & 70.85 \\
\hline 5 & $\{601,120\}$ & $\{621,125\}$ & 74.32 \\
\hline
\end{tabular}

By using Voila Jones algorithm we can achieve the good accuracy to detect the user's focus on monitor in $\mathrm{X}$ and $\mathrm{Y}$ coordinates. Voila Jones computes the image processing very faster than other techniques.

\section{ACKNOWLEDGEMENT}

For completing this project Prof. Anupkumar Bongal gave us important guidance. We are currently working on template matching of eye gaze detection using Voila Jones technique.

\section{REFERENCES}

[1]. W. Haibo, X. Chengqi, L. Qing, "The Eye Movement Experiment and the Usability Evaluation of the Fighter Cockpit Digital Interface," 2nd International Conf. Information Engineering and Computer Science (ICIECS) 2010, pp. 1-4, 2010. (10.1109/ICIECS.2010.5678205).

[2]. S. Milekic, "The more you look the more you get: Intention-based interface using gaze-tracking," in Trant, J.(Des.) Museums and the Web2002: Selected Papers from an Int. Conf., Archives and Museum Informatics, 2002, pp. 1-27.

[3]. J. Orozco, F. X. Roca, and J. Gonzàlez, "Real-time gaze tracking with appearance-based models," Machine Vision and Applications, 20(6): 353-364, 2009.

[4]. COGAIN: "D2.1 Survey of De-Facto Standards in Eye Tracking" by the European Commission within the Sixth Framework Programme, 2005.

[5]. Iannizzotto L. and La Rosa F., (2011) Competitive combination of Multiple Eye detection and Tracking Techniques, IEEE Transactions on Industrial Electronics, 58(8): 3151-3159.

[6]. Hansen D. W., Agustin J. S. and Villanueva A, (2010) Homography Normalization for Robust Gaze Estimation in Uncalibrated setups Proc. of the Symposium on Eye Tracking Research and Applications, pp. 13-20.

[7]. [7]. Javier Orozco, F. Xavier Roca and Jordi Gonzalez. (2009) Real time gaze tracking with appearance based models, Machine Vision and Applications, 20(6), 353364.

[8]. Lu F., Sugano Y., Takahiro O. and Sato Y., (2011) A head pose-free approach for appearance-based gaze estimation, in Proc. of the 22nd British Machine Vision Conf.

[9]. Zhu, Zhaomin, et al. "Multi-view face detection and recognition using haar-like features." Proceedings of COE Workshop. 2006.

[10].Dabhade, Sarala A., and Mrunal S. Bewoor. "Real Time Face Detection and Recognition using HaarBased Cascade Classifier and Principal Component Analysis." International Journal of Computer Science and Management Research 1.1 (2012).

[11].Padmaja, K., and T. N. Prabakar. "FPGA based real time face detection using Adaboost and histogram equalization." In Advances in Engineering, Science and Management (ICAESM), 2012 International Conference on, pp. 111-115. IEEE, 2012.

[12].Majumder, Anima, Laxmidhar Behera, and Venkatesh K. Subramanian. "Automatic and robust detection of facial features in frontal face images." InComputer Modelling and Simulation (UKSim), 2011 UkSim $13^{\text {th }}$ international Conference on, pp. 331-336. IEEE, 2011. 


\section{BIOGRAPHIES}

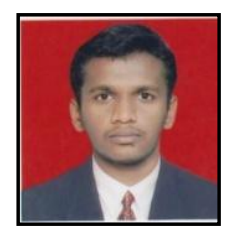

Mahadak Gopal Mahadeo

Gopalmahadak188@gmail.com(Bachelor

of Engineering Student, Computer Engineering Department, DY Patil College of Engineering, Ambi, Pune.)

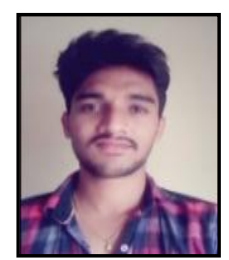

Landage Vijay Bapurao

Vijlandge1@gmail.com(Bachelor of

Engineering Student, Computer Engineering Department,DYPCOE, Ambi, Pune.)

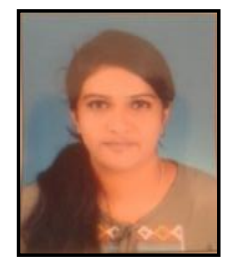

Solankar Rasika Sharad

solankarrs@gmail.com(Bachelor of Engineering Student, Computer Engineering Department, DY Patil College of Engineering, Ambi, Pune.)

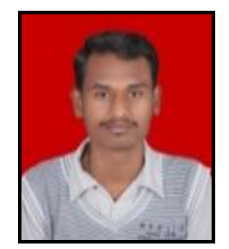

\section{Lohare Nitin Panditaro}

lohare45@gmail.com(Bachelor of

Engineering Student, Computer Engineering Department, DY Patil College of Engineering, Ambi, Pune.) 